\title{
Food Allergy Awareness among Parents of Food Allergic Child in Saudi Arabia
}

\author{
Nemer Alotaibi*, Lura Habib, Wejdan Alyamani, Renad Borah, Rakan Alquwayz, Bashar \\ Bin Nashar
}

Received: 27 September 2020 / Received in revised form: 08 December 2020, Accepted: 14 December 2020, Published online: 19 December 2020 (C) Biochemical Technology Society 2014-2020

(C) Sevas Educational Society 2008

\begin{abstract}
Food allergy is a worldwide challenge for children and their caregivers. Parent's awareness of food allergies is an important aspect to deal with reactions induced by food items. In Saudi Arabia data from 862 participants was gathered to assess their awareness about the child food allergy. The research was conducted by using an electronic questionnaire which was comprised of 27 different questions related to food allergy. All of the data was analyzed using SPSS. The results showed that few of the parents in Saudi Arabia were aware of food allergy but there was still an optimal need to deal with the disease condition rightly. Proper awareness of food allergies can prevent children from developing severe reactions.
\end{abstract}

Keywords: Food allergy, Anaphylaxis, Allergic awareness, epinephrine autoinjectors, Saudi Arabia

\section{Introduction}

Food allergy is a set of immunological responses that appears to happen when a person shows a certain type of interaction with some food items (Bollinger et al., 2006; Phan et al., 2020). In the past few years, several research studies were conducted which delineated that children ranging from age birth to 15 are more likely to get allergic diseases including food allergy (Mahmoud $e t$ al., 2020; Zewde, 2020). prevalence of true food allergies in children range from 6-8\% in united stat (Gupta et al., 2011) the prevalence in united Arab emirate was reported the same as for western countries (8\%)(Al-Hammadi et al., 2010; Al-Herz, 2018), Although No clear data about the prevalence of true food allergy in Saudi Arabia, several studies reported the food sensitivity

Nemer Alotaibi *

Assistant Professor of Pediatrics, Department of Pediatrics, College of Medicine dawadmi, Shaqra University, Shaqra, Saudi Arabia.

Lura Habib

Collage of medicine, Taif University, Taif, Saudi Arabia.

Wejdan Alyamani, Renad Borah

Collage of Medicine, Batterjee Medical College, Jeddah, Saudi Arabia.

Rakan Alquwayz, Bashar Bin Nashar

Collage of medicine, Shaqra University, Shaqra, Saudi Arabia.

*E-mail: Dr.nemeralotaibi @ hotmail.com among children suffering from other allergic diseases to reach up to $29 \%$. (Aba-Alkhail and El-Gamal, 2000).

Several symptoms could indicate the presence of food allergy and these symptoms are usually mild but sometimes can lead to severe reactions and might cause life-threatening events, sever reaction could present in up to $38.7 \%$ of children (Gupta et al., 2011) reactions could affect different sites including Skin cardiovascular, gastrointestinal and respiratory tract. Anaphylaxis is the most severe life-threatening reaction. In anaphylaxis, the time for the onset of reaction is just a few minutes after the ingestion of food (S H Sicherer et al., 2000; Alqahtani et al., 2020). It can cause a fatal reaction and should be treated immediately with adrenaline then transferred to a medical facility. In children, food allergy is majorly caused by foods such as milk, eggs, wheat, peanuts, tree nuts, soy, fish, shellfish, $30 \%$ of food-allergic children have multiple food allergies, and the most common food allergen in the west is peanuts followed by milk and shellfish (Gupta et al., 2011). It is commonly presented with rash, hives, swelling, tingling of lips and tongue, vomiting, and in some cases breathing difficulty and anaphylactic shock. (Scott H Sicherer and Sampson, 2010)

The only existing therapy for food allergy is the avoidance of the food allergen (Boden and Wesley Burks, 2011; Scott H Sicherer and Sampson, 2010). However, avoidance can be a challenge because many of the most common allergens are prevalent in many food items, such as milk, eggs, peanuts, tree nuts, seafood (such as fish and shellfish), sesame, soy, and wheat (Kagan, 2003).

Food allergy during childhood also affects parents and the family as a whole (Bollinger et al., 2006). Parents must have adequate disease knowledge to accurately understand the sources of allergens, risks, severity, and avoidance methods. Having accurate knowledge may also increase the confidence of the parents to treat their child immediately without fears about harming the child when administering the injection (Hahn et al., 2017).

Food allergy is not just confined to children, it affects the whole family as well. For this fact, the parents must possess enough knowledge and awareness of allergic conditions. This awareness can significantly play a crucial role in the prevention of children from getting severe food reactions. Having appropriate and right knowledge for dealing the allergic conditions might also elevate the confidence of parents to treat the affected child immediately without even drawn to certain fears while administration. (Gupta et al., 2008) 
It is estimated that accidental exposures occur in $50 \%$ of children with food allergy(Wood, 2003) Consequently, parents must be familiar with symptoms and trained in how they should respond to reactions, including proper usage of epinephrine auto-injectors and utilization of emergency services. However, several parents demonstrated fear of using epinephrine auto-injectors or incorrect use of the auto-injector and appeared unaware of the need to replace expired auto-injectors. (Bock et al., 2007)

This study was conducted to assess the potential awareness of parents regarding food allergies in Saudi Arabia. The study was carried out based on an analysis in the form of survey forms. This study also explains the potential aspects of food allergy.

\section{Materials and Methods}

\section{Research Design}

An observational cross-sectional electronic questionnaire-based study was consistent with 27 well-formed questions concerning the awareness of parents about their food allergies in children. The questionnaire was distributed electronically through social media platforms. Level of awareness was assessed in three main domains which includes the knowledge of food allergy symptoms for both parents with allergic child, background information about food allergy, and management of food allergy, for each domain we asked specific questions, for symptoms we asked the general public parents to give their opinion about which food allergy symptoms they think could happen to the allergic child, we asked the parents if they know before about food allergy , and whether the food allergy is secondary to immunological reaction or not, for the management part we assess the awareness of parents of allergic child only by asking the parents if they seek medical advice in case of allergic reaction or not, which medication was given, if their child underwent food allergy testing or not, if everyone deals with the child at home knows about his food allergies, if the school or kindergarten teachers and workers knows about their food allergy or not, and finally if they wear allergy identification or not .

Data analysis was carried out using the statistical package for social sciences version 23 (SPSS Inc, Chicago, IL, USA) for further conclusions.

\section{Participants, Sample Size and Selection}

The study took place among the general population of people of a specific age group living in different parts of Saudi Arabia. The criteria included all Saudi and non-Saudi male and female children who have a certain food allergy, children up to 15 years of age. A simple random sampling technique was used to select the participants randomly from the large population of Saudi Arabia. However, those aged above 15 years of age and parents not willing to participate are excluded. With the help of the participants' parents and guardians, the researcher was able to get access to a total of 862 respondents in the study. The target respondents were parents and guardians of children suffering from a food allergy and aged between birth to 15 years only.

\section{Data Collection methods}

An online questionnaire was used to collect the data for the study. questionnaire contain multiple choices and closed ended-questions translated to Arabic for better understanding by the respondents. A link was created that would enable the respondents to access the questionnaire through social media and respond to the prepared questions. The use of an online questionnaire was preferred due to the COVID 19 pandemic that requires people to keep social distance and hence, would ensure safety for both the researcher and the respondents. The respondents were then able to give their views concerning the parents' awareness and dealing with their foodallergic child in Saudi Arabia.

\section{Data Analysis Plan}

The collected data was analyzed using SPSS. The quantitative analysis involved descriptive statistics and a chi-square test. The descriptive statistics were done to give frequencies of the responses given on various questions. The chi-square test on the other hand was done to check for the existence of any relationship between the related variables. It was also to respond to the research question and hypothesis. Additionally, both the descriptive statistics and the inferential statistics would confirm whether the research objectives were met accordingly. The obtained chi-square significant value was compared with the alpha value of .05 on whether to reject or accept the null hypothesis at a $95 \%$ level of significance.

\section{Ethical consideration}

A document of consent was sent to the participants before the administration of the research questionnaire. The document briefed participants on the reasons for collecting data and the objectives of the study. Responding to the questions was voluntary and only those who were willing were allowed to participate in the study. Brief instructions were stated on the questionnaires which did not allow the participants to include any of their personal information on the questionnaires. They were also assured that their views would remain confidential and would not be accessed by any third party other than the researcher. The study was approved by the IRB of the research institution.

\section{Results}

In this section, the sample description will be discussed of the demographic variables of the participants of the study.

\section{Sample Description}

In this section, the percentage and frequency distribution of the demographic characteristics of the participants in the current research $(\mathrm{N}=862)$ is described. There was a total of six demographic variables and these were gender, nationality, region, education, socio Economic Status and no. of children. 
Table 1: Frequencies and Percentages of Demographic variables of Participants $(\mathrm{N}=862)$

\begin{tabular}{|c|c|c|}
\hline Demographics & $\mathrm{f}$ & $\%$ \\
\hline \multicolumn{3}{|l|}{ Gender } \\
\hline Male & 286 & 33.2 \\
\hline Female & 563 & 65.3 \\
\hline \multicolumn{3}{|l|}{ Nationality } \\
\hline Saudi & 820 & 95.1 \\
\hline Non-Saudi & 42 & 4.9 \\
\hline \multicolumn{3}{|l|}{ Region } \\
\hline Central & 185 & 21.5 \\
\hline Eastern & 274 & 32.1 \\
\hline Western & 194 & 22.5 \\
\hline Northern & 190 & 22.0 \\
\hline Southern & 16 & 1.9 \\
\hline \multicolumn{3}{|l|}{ Education } \\
\hline Less than high school & 27 & 3.1 \\
\hline High school & 142 & 16.5 \\
\hline Bachelor & 609 & 70.6 \\
\hline Postgraduate & 84 & 9.7 \\
\hline \multicolumn{3}{|l|}{ Socio Economic Status } \\
\hline Low & 61 & 7.1 \\
\hline Medium & 725 & 84.1 \\
\hline High & 76 & 8.8 \\
\hline \multicolumn{3}{|l|}{ No. of children } \\
\hline Less than 6 & 767 & 89.0 \\
\hline More than 6 & 95 & 11.0 \\
\hline
\end{tabular}

Note. $f=$ Frequency, $\%=$ Percentage

As indicated in table 1, the sample size was 862 out of which 286 were male participants $(33.2 \%)$ and 563 were women participants (65.3\%). The table also indicated that out of 862 participants $(\mathrm{N}=$ $862)$ there were $820(95 \%)$ participants were Saudi national while $42(4.9 \%)$ participants were non-Saudi national. The sample was consisting of $185(21.5 \%)$ participants were from the central region, $274(32.1 \%)$ from the eastern region, 194 (22.5\%) from the western region, $190(22.0 \%)$ from the northern, and $16(1.9 \%)$ from the southern region. The sample belonged to less than high school consists of 27 (3.1\%), high school 142 (16.5\%), bachelor consists of $609(70.6 \%)$ and postgraduate $84(9.7 \%)$. The sample consists of $61(7.1 \%)$ participants who had low socio-economic statuses while $725(84.1 \%)$ had medium socio-economic and $76(8.8)$ were have high socioeconomic status. In our survey, most of the children are less than 6,767 age with the $89.0 \%$, and the number of children more than 6,95 are $11.0 \%$.

\section{Graphical representation of demographic variables}

\section{nationality}

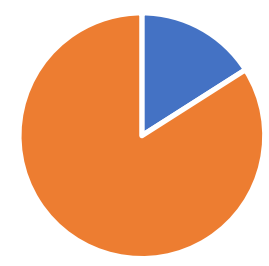

- less than high school " high school

Figure 1. nationality of the particepant

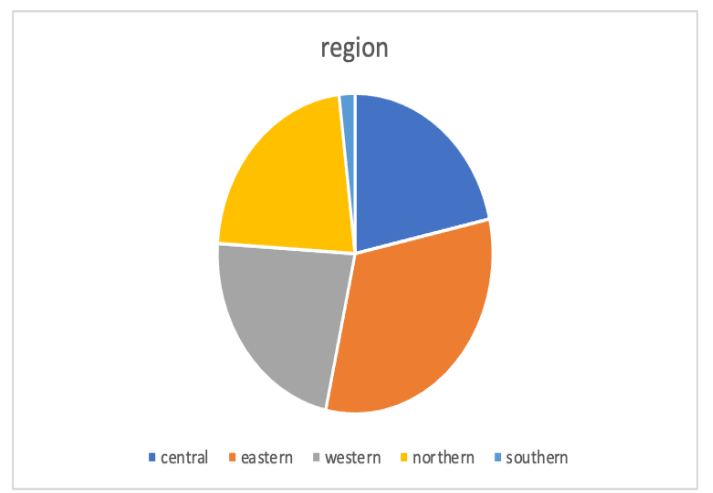

Figure 2. distribuation of particpant

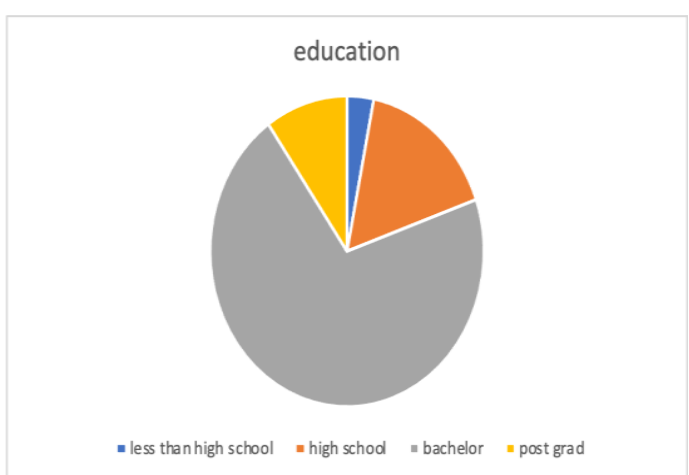

Figure 3. level of education of parents

Figure number (1) shows that the majority of participants were Saudi nationals, while figure number(2) represents that participants were from different areas (southern, northern, eastern, central, western) of Saudi Arabia and it was observed that a majority of people were from eastern area, northern, central, western areas showed an equal number of participants and there was a very little contribution from southern area, On the other hand, figure number (3) highlights that most of the participants were well educated and the majority of them had a bachelor qualification.

In the current study, 862 participants were asked questions about the awareness of food allergies. Descriptive tables and graphical representation revealed the awareness of the participants 
Table 2: Food Allergy Awareness

\begin{tabular}{|c|c|c|c|c|c|c|}
\hline Questions & Agree & Frequency & Disagree & Frequency & t'noD wonk & Frequency \\
\hline Is food allergy an abnormal immune response to food? & $84.8 \%$ & 731 & $3.2 \%$ & 28 & 11.9 & 103 \\
\hline $\begin{array}{c}\text { Does your child have a diagnosis of food allergy from a } \\
\text { healthcare provider? }\end{array}$ & $22.5 \%$ & 194 & $77.5 \%$ & 668 & I & I \\
\hline $\begin{array}{l}\text { Have blood tests or skin prick been done to determine the cause } \\
\text { of food allergy? }\end{array}$ & $12.2 \%$ & 105 & $32.3 \%$ & 278 & $55.6 \%$ & 479 \\
\hline Is there medication prescribed for the condition? & $20.3 \%$ & 175 & $43.4 \%$ & 374 & $36.3 \%$ & 313 \\
\hline Does your child know she or he has this allergy? & $19.8 \%$ & 171 & $32.8 \%$ & 283 & $47.3 \%$ & 408 \\
\hline Does the child have a caregiver other than his mother? & $22.3 \%$ & 192 & $77.7 \%$ & 670 & 1 & 1 \\
\hline $\begin{array}{l}\text { If your child goes to kindergarten or school, do workers in that } \\
\text { facility know about your child's food allergy type? }\end{array}$ & $16.6 \%$ & 143 & $30.6 \%$ & 264 & $11.6 \%$ & 100 \\
\hline $\begin{array}{l}\text { Does your child wear a medical alert bracelet or necklace to } \\
\text { make people aware of his or her food allergy? }\end{array}$ & $2.2 \%$ & 19 & $57.9 \%$ & 499 & $39.9 \%$ & 344 \\
\hline
\end{tabular}

Total Participants $=862$

As showen in table \# 2 most of general population knows about nature of food allergy as $84 \%$ agreed it is secondary to immunological respoce towards food , $22.5 \%$ had official diagnosis from healthcare providers .

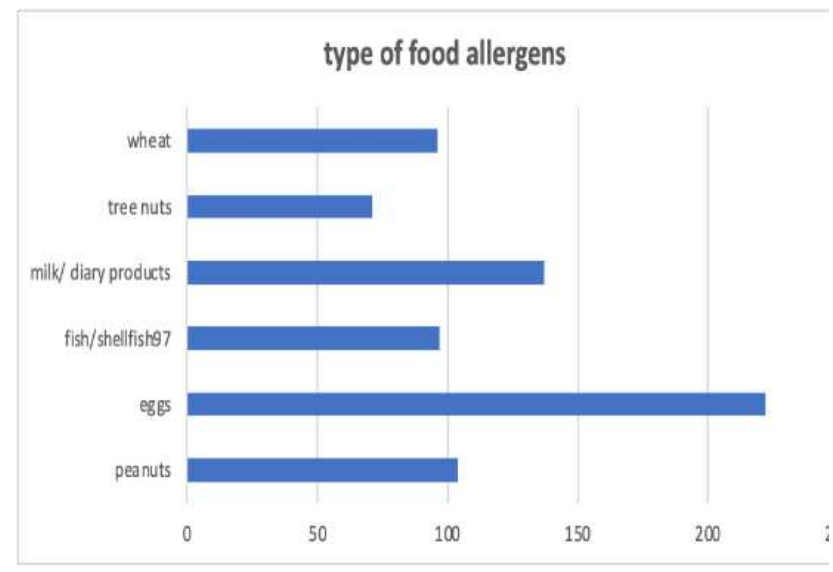

Figure 4. type of food allergen
The graphical representation of the bar chart in figure \# 4 above revealed that the majority of allergic reactions were secondary to eggs 222 (46\%), second most common food allergen in our survey are cows milk and dairy products $137(28 \%)$, peanuts $104(21 \%)$, wheat 96(20\%), fish shellfish (20\%), tree nuts 71(14\%).

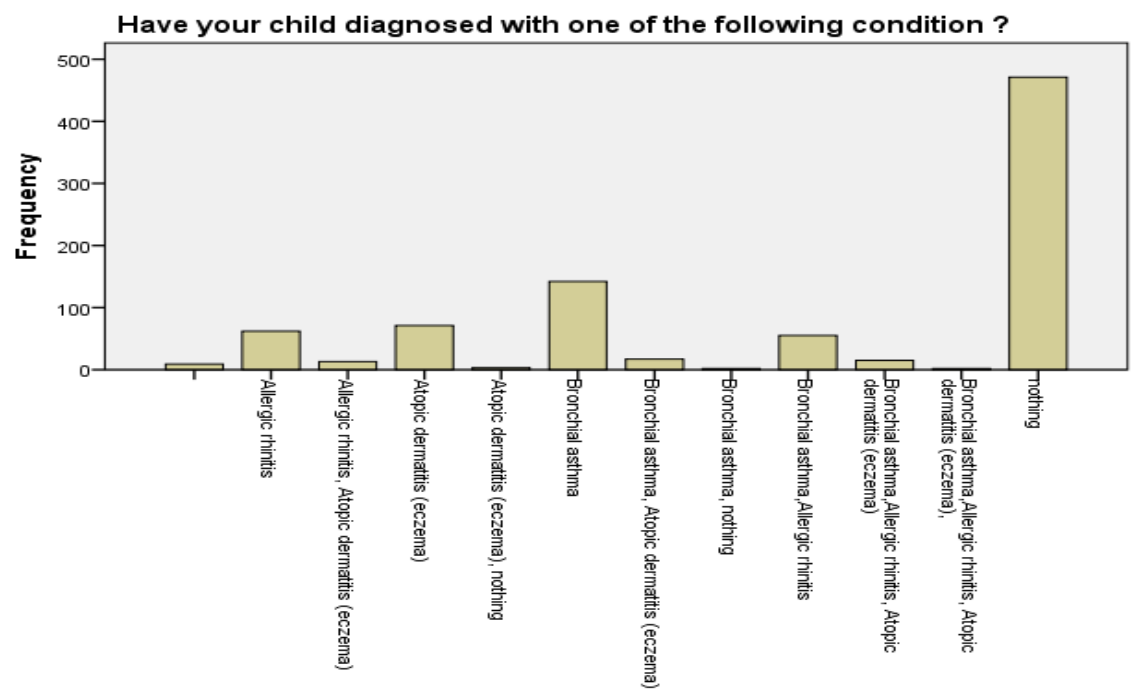

Figure 5. co-morbid disease 
The bar graph in figure \# 5 above shows that the majority of the participants had no comorbid allergic or atopic disease. Of the atopic disease, the most prevalent in our study was bronchial asthma followed up by atopic dermatitis and allergic rhinitis

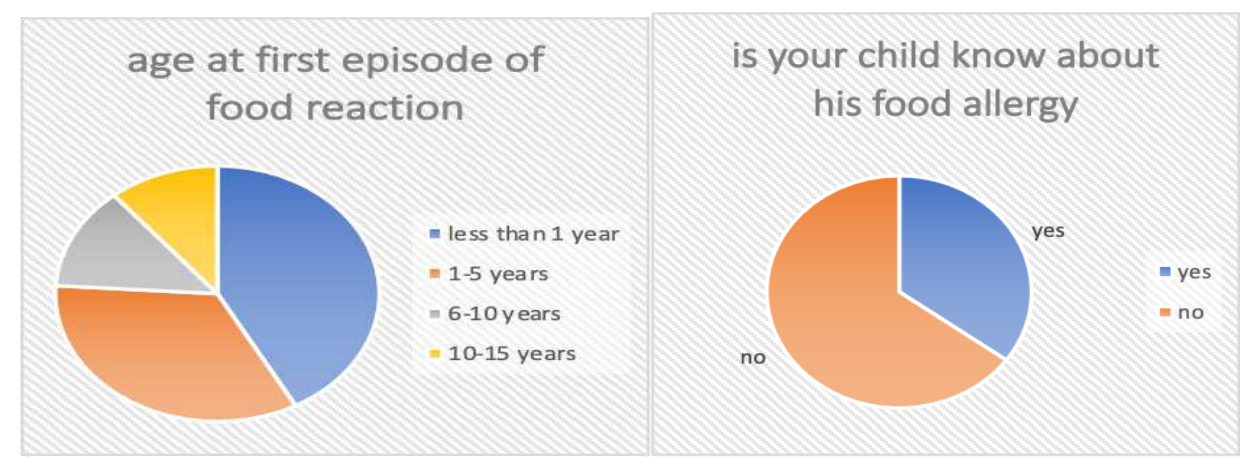

Figure 6. age of first reaction, figure \# 7 child awareness of food allergy

The pie chart in figure \#6 represents the age at which the first food reaction happened. The majority of our surveyed children had reactions before the age of five years $(77 \%)$, and (10\%) had reactions between the age of 5-10 years. The remaining (13\%) had reactions after the age of 10 years. In figure (7), most of the parents reported that children do not know about their food allergy (65\%). The remaining (35\%) reported they know about the types of food allergies.

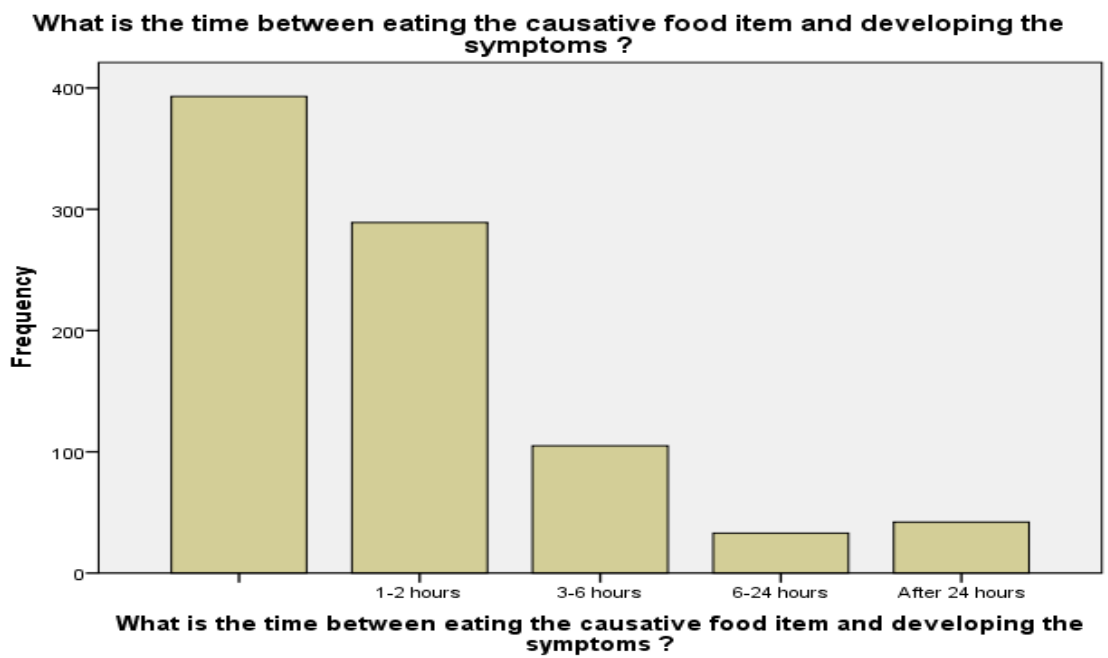

Figure 8. timing of reaction symtpomes

The bar chart above in figure \# 8 shows that majority of the participants did not respond to this question. 289 of them have found symptoms after 1-2 hours of eating. While 105 have found the symptoms after 3-6 hours of eating and 33 participants get the symptoms after 8-24 hours of eating. 


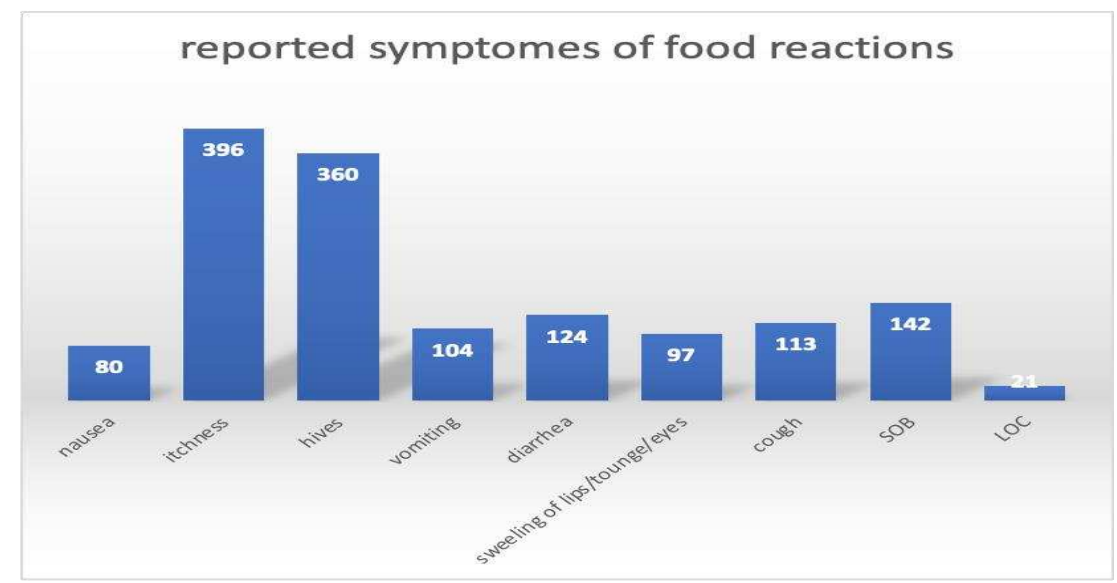

Figure 9. reported symtpomes of food reactions

The bar graph in figure \# 9 above shows that majority had Skin manifestations such as itchiness $396(82 \%)$ and hives 360 (74\%) were the most reported symptoms by parents. Respiratory symptoms include cough $113(23 \%)$ shortness of breath 142 (29\%), followed by GI manifestation, diarrhea 124 (25\%) vomiting $104(21 \%)$ nausea $80(16 \%)$, and loss of consciousness in $21(4 \%)$.

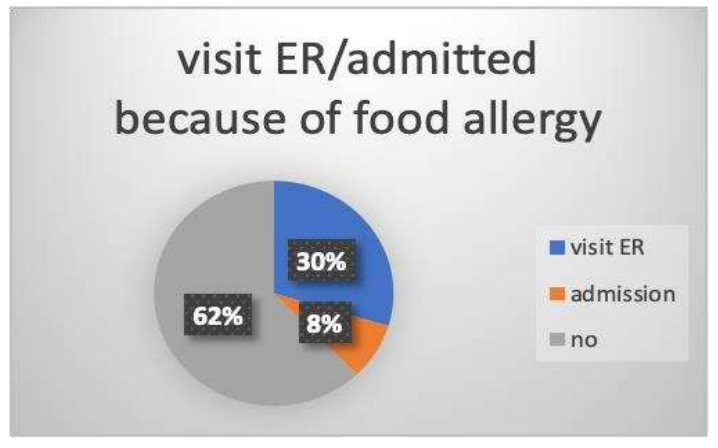

Figure 10. seek medical advise

figure \# 10 represents how many parents seek medical advice after the first food reaction. Only $38 \%$ visited medical facilities Out of the $38 \%, 30 \%$ visited the emergency room and $8 \%$ got admitted to the hospital. $62 \%$ denied visiting medical facilities.

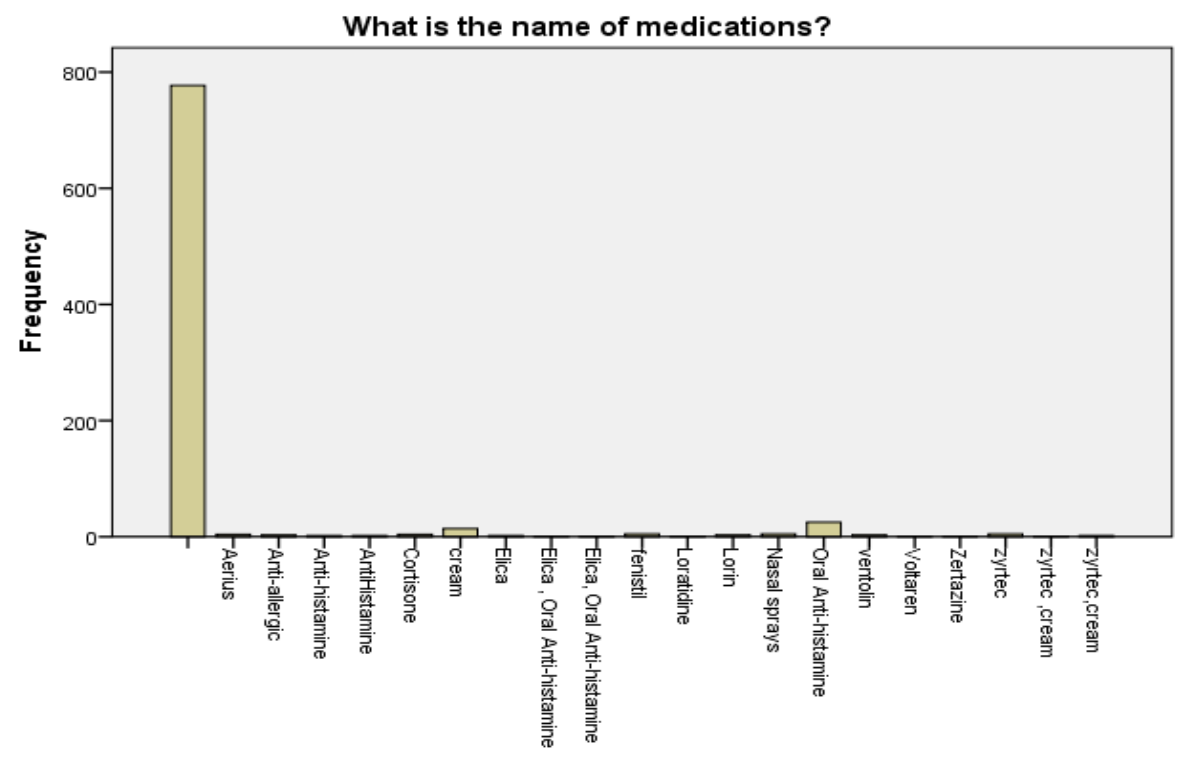

What is the name of medications?

Figure 11. medication prescribed 
The graphical representation in figure \# 11 above showed that the majority of the participants did not respond to this question. 25 of them talk about Oral Anti-histamine. While 14 participants said that they know about cream medication.

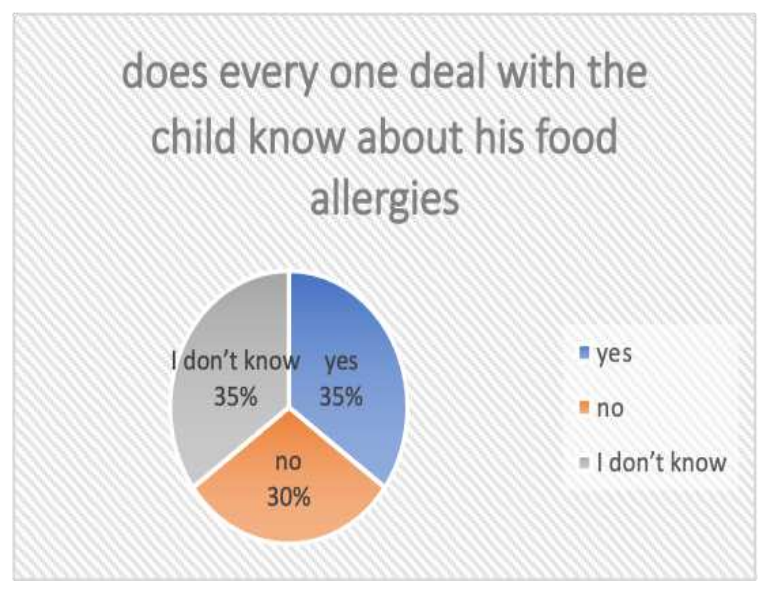

Figure 12. awarness of household/teachers

Figure (12) represents how everyone dealing with the allergic child are aware of his food allergy As shown, only $35 \%$ are aware of his food allergy condition, $30 \%$ are not aware, and 35\% of the parents do not know if others are aware of their child food allergies.

\section{Discussion}

Food allergy is an increasing issue with more persistence in its occurrence from the past few years. This research study was significant as a fact that the majority of parents with allergic kids had little awareness about child food allergy management. The reaction resulting from the food allergy range from mild to severe, and in some cases, it develops into a life-threatening form. The best and only optimal way to avoid the development of allergic reactions is the complete avoidance of the food items to which one is allergic. To prevent the child from developing any kind of food allergy, there is a strong need to be aware of the necessary protocols to be followed. The parents should possess enough knowledge to deal with different allergic conditions of their children and must also be able to deal with difficult challenges in the treatment of disease.

The prevalence of food allergy in children is estimated to be around 8 percent in the United States and Arabian Peninsula (Gupta et al., 2011; Al-Hammadi et al., 2010; Al-Herz, 2018). Although there is no clear data about the prevalence of true food allergy in Saudi Arabia, several studies reported that the food sensitivity among children suffering from other allergic diseases reaches up to $29 \%$ (Aba-Alkhail and El-Gamal, 2000). In our survey, almost 50\% of the parent responders reported some sort of food reaction. This could be because we targeted parents with children suffering from food allergic reactions. This study was conducted in different areas of Saudi Arabia, one-third of the responders come from the eastern province. The food allergy diagnosis rate was higher in the western province $37 \%$ compared to only $11 \%$ in the eastern province. The central, northern, and southern providence showed a diagnosis rate of $28 \%, 25 \%, 25 \%$ respectively. That means only around a quarter of food allergic children received the official diagnosis of food allergy. This may be due to the shortage of specialized allergists or the inability of patients to access tertiary hospitals in these areas. Another possibility may that responders living in rural versus urban areas have been shown that regional variations may exist in the rate of diagnosis of food allergy. (Botha et al., 2019).

The most common food allergen reported in children is milk, eggs, wheat, nuts, and peanuts. In our study, the most reported food reactions following the ingestion of eggs in $46 \%$ followed by milk $28 \%$ then peanuts $22 \%$, and wheat $20 \%$, Only 5 patients reported sesame allergy. This study has found that some of the foods causing allergy especially to children include egg and milk which usually affect the child who is being introduced foods. (Pitchforth et al., 2011) our data showed the eggs and dairy products are frequent causes of food allergy in young children's similar to reports from the UAE (Al-Hammadi et al., 2010) compared to the united states in which peanuts are the most frequent causes of food allergies. (Scott H Sicherer, 2011; Gupta et al., 2011; Kagan, 2003).

the most reported symptoms by parents include skin manifestation with itchiness and hives in $67 \%$ and $61 \%$ respectively. The cough was reported in $19 \%$, difficulty in breathing $24 \%$ GI manifestations comes after with vomiting and diarrhea in $19 \%$ and $21 \%$, which compatibles with other studies (Scott H Sicherer, 2011; Kagan, 2003; Scott H Sicherer and Sampson, 2010), the clinical manifestation of anaphylaxis in our survey reported in almost $4 \%$. almost $70 \%$ of the symptoms occurred in the first 2 hours after ingestions and $30 \%$ after 3 hrs. with only $7 \%$ after $24 \mathrm{hrs}$. which is typical for IGE mediated reactions.

One of the most important predictors of parents' awareness in dealing with food-allergic children is that everyone who deals with the child should know about the type of food allergen and how to deal with food allergy symptoms and reactions. In our study, only $35 \%$ of parents with an allergic child reported that everyone dealing with their child knows about his food allergy whereas $65 \%$ reported do not. These finding compatibles with Some studies report defect knowledge about food allergy among nannies (Posner and Camargo, 2017).

School personnel should be aware of the possibility of food reactions inside the school and epinephrine auto-injectors should be available, written action plan along with prefilled epinephrine auto-injectors should be offered to all school-age children suffering from a food allergy (Greiwe et al., 2015).

Some of the ways that indicate awareness about food allergy includes; provision of caregiver to the allergic child other than the mother, both the parents and the allergic child should be aware of the child's allergic condition and there should also be medication prescription for the child among other factors (Wood, 2003).this study has found that the majority of kids are not aware of their food allergy and that most probably because in our study most of the children reported are less than 5 years of age and they are not aware of the meaning of food allergy. 
Most of the parents did not seek medical advice in our study and only $20 \%$ received an official diagnosis of food allergy from allergy specialists. Therefore, do not consider visiting specialized medical personnel to evaluate the possibility of a food allergy. This is a clear indication that both the parents and the allergic children in Saudi Arabia are not well informed about the food allergic condition and hence, there is a need to create more awareness about food allergy in Saudi Arabia.

When it comes to medication, the majority of parents said that no medication was prescribed and some people were used certain creams to deal with the allergic condition. Studies showed that most of the children with food allergies were treated with oral antihistamine which is compatibles with our current study in which most parents reported using oral or topical antihistamine plus local steroid creams. In our survey, only one patient reported a prescription of autoinjector epinephrine, which is a mandatory medication for patients suffering from IGE mediated reactions especially those who were present previously in an emergency department or got admitted. It was proven that delay use of epinephrine or inappropriate use results in increase risk and mortality of food allergic reactions. (Gupta et al., 2018) Adding to the low prescription rate of epinephrine auto-injectors, studies showed that some parents may be reluctant to give the epinephrine or had incorrect use or even carry expired medication (Bock et al., 2007)

Although most of the parents have strong educational background i.e. more than $80 \%$ of the participants have a bachelor's qualification. The study did not establish a statistically significant association between the parents' level of education and the level of awareness, which was shown in other studies. (Bollinger et al., 2006), Again, there is no significant association between the gender of the child or gender of parents and the level of awareness The findings in our study agrees with a study done among undergraduate students in Ghana (Adegbiji et al., 2020).

The study revealed that out of a total of 862 participants $84.8 \%$ or 731 participants agreed that food allergy is an abnormal response that is caused as a result of interaction with certain food items. This showed that most of the people had enough awareness about the nature and symptoms of food allergies.

A medical ID is used for the identification of a specific type of diseased patient and it was found from the study that 499 people did not use medical id (bracelets, chains, tags, wristbands), 344 participants did not have any idea about the medical id and its purpose and only 19 people i.e. $2.2 \%$ of the total participants were using the medical id for their children. This shows little awareness about the importance of allergy identifications especially for kids who cannot communicate properly as suggested by several allergy guidelines.

This study had a couple of limitations. The study was entirely done using electronic channels given the covid-19 situation. As a result, the study had little control over the responses. Also, the study is concentrated in one region and hence, the generalization of results is a bit limited.
The best way to prevent children from a food allergy is by avoiding their exposure to the food items to which they are allergic. This can be only possible when the parents of the children will have awareness regarding the food allergies and how to prevent them from developing. Several health training camps can be organized weekly to give the relevant and right knowledge about the protocols to be followed under the disease influence and in this way food allergy reactions can be effectively reduced.

\section{Conclusion}

Food allergy is a major issue for children up to the age of 15 years. This study showed that a majority of people in Saudi Arabia were little aware of child food allergies. It was also seen that most of them did not know about the medical id and symptoms of the disease. Moreover, some showed no interest in taking their allergic child to visit the ER or hospital. The proper awareness about the reaction can help in the reduction of food allergies and prevent the child from developing any reactions. The onset of food allergies can be minimized by avoiding the use of food items one is allergic to. Moreover, the relevant knowledge regarding food allergy in children can help the parents to deal with different allergic conditions.

\section{References}

Aba-Alkhail, B. A., \& El-Gamal, F. M. (2000). Prevalence of food allergy in asthmatic patients. Saudi medical journal, 21(1), 81-87.

Adegbiji, W. A., Aremu, S. K., Aluko, A. A. A., \& Adewoye, R. K. (2020). Knowledge and awareness of nasal allergy among patients in a developing country. Journal of Family Medicine and Primary Care, 9(3), 1477.

Al-Hammadi, S., Al-Maskari, F., \& Bernsen, R. (2010). Prevalence of food allergy among children in Al-Ain city, United Arab Emirates. International archives of allergy and immunology, 151(4), 336-342.

Al-Herz, W. (2018). A systematic review of the prevalence of atopic diseases in children on the Arabian Peninsula. Medical Principles and Practice, 27(5), 436442.

Alqahtani, A. N., Alanazy, S. H., Aljameel, O. S. H., Aldhawi, F. S. M., Jamjoom, M. M., Al Sharhan, A. H., ... \& Salman, I. M. (2020). Evaluation of Diagnosis and Management of Anaphylactic shock in Emergency Room: A Literature Review. International Journal of Pharmaceutical Research \& Allied Sciences, 9(1).

Bock, S. A., Muñoz-Furlong, A., \& Sampson, H. A. (2007). Further fatalities caused by anaphylactic reactions to food, 2001-2006. Journal of Allergy and Clinical Immunology, 119(4), 1016

Boden, S. R., \& Wesley Burks, A. (2011). Anaphylaxis: a history with emphasis on food allergy. Immunological reviews, 242(1), 247-257.

Bollinger, M. E., Dahlquist, L. M., Mudd, K., Sonntag, C., Dillinger, L., \& McKenna, K. (2006). The impact of food allergy on the daily activities of children and their 
families. Annals of Allergy, Asthma \& Immunology, 96(3), 415-421.

Botha, M., Basera, W., Facey-Thomas, H. E., Gaunt, B., Gray, C. L., Ramjith, J., ... \& Levin, M. E. (2019). Rural and urban food allergy prevalence from the South African Food Allergy (SAFFA) study. Journal of Allergy and Clinical Immunology, 143(2), 662-668.

Greiwe, J. C., Pazheri, F., \& Schroer, B. (2015). Nannies' knowledge, attitude, and management of food allergies of children: an online survey. The Journal of Allergy and Clinical Immunology: In Practice, 3(1), 63-67.

Gupta, R. S., Kim, J. S., Barnathan, J. A., Amsden, L. B., Tummala, L. S., \& Holl, J. L. (2008). Food allergy knowledge, attitudes and beliefs: focus groups of parents, physicians and the general public. BMC pediatrics, 8(1), 36 .

Gupta, R. S., Springston, E. E., Warrier, M. R., Smith, B., Kumar, R., Pongracic, J., \& Holl, J. L. (2011). The prevalence, severity, and distribution of childhood food allergy in the United States. Pediatrics, 128(1), e9-e17.

Gupta, R. S., Warren, C. M., Smith, B. M., Blumenstock, J. A., Jiang, J., Davis, M. M., \& Nadeau, K. C. (2018). The public health impact of parent-reported childhood food allergies in the United States. Pediatrics, 142(6)..

Hahn, A. L., Dahlquist, L. M., Hoehn, J. L., \& Elizabeth Bollinger, M. (2017). Development of a food allergy knowledge test for parents. Journal of pediatric psychology, 42(5), 598609.

Kagan, R. S. (2003). Food allergy: an overview. Environmental health perspectives, 111(2), 223-225.
Mahmoud, M. H., Wahba, H. M. A., \& Mahmoud, M. H. Investigating a baby food prepared from Opuntiaficusindica in Wistar rats.

Phan, N. H. D., Nguyen, T. T., Tran, T. B. H., Vo, N. T., Le, T. T. T., Quang, M. T., ... \& Lao, T. D. (2020). EXPLORING THE PCR ASSAY FOR DETECTING TROPOMYOSIN: MAJOR ALLERGEN IN SHRIMP-DERIVED INGREDIENT IN FOOD. Pharmacophore, 11(2).

Pitchforth, E., Weaver, S., Willars, J., Wawrzkowicz, E., Luyt, D., $\&$ Dixon-Woods, M. (2011). A qualitative study of families of a child with a nut allergy. Chronic illness, 7(4), 255-266.

Posner, L. S., \& Camargo Jr, C. A. (2017). Update on the usage and safety of epinephrine auto-injectors, 2017. Drug, healthcare and patient safety, 9, 9.

Sicherer, S. H. (2011). Epidemiology of food allergy. Journal of Allergy and Clinical Immunology, 127(3), 594-602.

Sicherer, S. H., \& Sampson, H. A. (2010). Food allergy. Journal of allergy and clinical immunology, 125(2), S116-S125.

Sicherer, S. H., Forman, J. A., \& Noone, S. A. (2000). Use assessment of self-administered epinephrine among foodallergic children and pediatricians. Pediatrics, 105(2), 359362.

Wood, R. A. (2003). The natural history of food allergy. Pediatrics, 111(Supplement 3), 1631-1637.

Zewde, G. T. (2020). Assessment of Knowledge, Attitude, and Practices of Colostrum Feeding among Postnatal Mothers in Harar Town Governmental Hospital Harar, Ethiopia 2019. Journal of Pedaitrics Research Reviews \& Reports. SRC/JPRRR-107, 3. 